\title{
The Design and Implementation of Collaboration Service Integration Platform Based on Context-Aware Role Based Access Model
}

\author{
Shu-Ping Lu ${ }^{1}$, Kuei-Kai Shao ${ }^{1}$, Yu-Nung Chao ${ }^{1}$, \\ Kuo-Shu Luo ${ }^{1}$ and Chi-Hua Chen ${ }^{1,2,3}$ \\ ${ }^{1}$ Service Systems Technology Center, Industrial Technology Research Institute, \\ Hsinchu, Taiwan, R.O.C. \\ ${ }^{2}$ Institute of Information Management, National Chiao Tung University, \\ Hsinchu, Taiwan, R.O.C. \\ ${ }^{3}$ Telecommunication Laboratories, Chunghwa Telecom Co., \\ Ltd, Taoyuan, Taiwan, R.O.C. \\ dolinlu@itri.org.tw; davidshao@itri.org.tw; rachelchao@itri.org.tw; \\ JonesLo@itri.org.tw; chihua0826@gmail.com
}

\begin{abstract}
In recent years, the effective management of organization's business processes has become more and more important to support the business workflows. Although some collaboration platforms have been created to support business processes, the user and role authorities in these platforms cannot automatically change in accordance with context information (e.g., time and location, and environment information). Therefore, this study designs and implements the Collaboration Service Integration Platform (CSIP) which combines Business Process Model and Notation (BPMN) and Context-Aware Role-Based Access Control (CARBAC) model. The context information is considered to enhance RBAC and adjust the authority of user role dynamically. For implementation, the logic gateways and connectors are added in the tasks and workflows for adjusting the authority dynamically. The right information can be accessed by the right user in CSIP based on CA-RBAC.
\end{abstract}

Keywords: Access Control Model, Business Process Model and Notification, Role-Based Access Control

\section{Introduction}

With the globalization of markets, firms have struggled to achieve greater collaboration to leverage the knowledge and resources [1]. The effective management of organization's business processes, as a result, became ever more important [2-4]. Fundamentally, business processes either private or public business processes commonly found in business organizations or inter-organizational collaboration. Well business management for a series of tasks for different workflows of jobs or processes within an organization can make production and services more efficient. Interfirm collaborations with efficient business management instruments can improve the competitiveness of companies. Handle processes with tasks and data separated by enterprise boundaries, however, is hard for process collaboration environments, for reasons such as security, privacy, etc [5].

Google Sites is a popular collaboration platform which provides highly configurable blog, Wiki and content management functions [6]. Users can create and share contents with each other on the web. A collection of group documents interactively is distributed and processed on the site. Google site can streamline workflows and allow everyone to work together. 
Nevertheless, Google Sites is not ideal because of signoff without watermark, lack of workflow agent listener service, limited use of HyperText Markup Language (HTML) coding and lack of Cascading Style Sheets (CSS) incorporated in the theme templates, etc [6]. To avoid the limitations of business processes in Google Sites, this study develops collaboration service integration platform which is combined with open-source business process management and workflow suites.

In order to guarantee that the protected business services can only be accessed by authorized users as the specified manners in collaborative environment, access control [7] is an important authorization policy and security mechanism for organizations to protect their resources in collaborative environments [8]. Rule-Based Access Control (RBAC) [9] model is practical and widely deployed in distinct enterprises. Furthermore, Context-Aware RBAC (CA-RBAC) [10] can involve the contextual attributes such as location and time. Therefore, this study proposes the Collaboration Service Integration Platform (CSIP) using CA-RBAC to satisfy the increasing demands on the different contextual attributes. The CSIP is based on Business Process Model and Notation (BPMN) 2.0 which is an important standard and supplied by large global software companies.

The remainder of this study is structured as follows. In Section 2 this study provides background knowledge through the description of related technologies, such as the concept of access control models. In Section 3 this study designs and implements the architecture of CSIP. A case study of CSIP is given for Online Authorization (OA) in Section 4. Finally, conclusions and future work are given in Section 5.

\section{Related Work}

To produce the description to reality is the main objective in business model. As a consequence, notations to allow us model the essence of business as clearly as possible are important [11]. The Business Process Model Notation (BPMN) can express in a graphical fashion at the business process modeling level which in turn can be transformed into corresponding access control for process-aware information systems.

\subsection{Access Control Models}

Previous research specifying the different access control policies has presented dozens of access control models since the late 1960s'. The research community contains Access Matrix [12], Discretionary Access Control (DAC) [13], Mandatory Access Control (MAC) [13], and RBAC [14]. While DAC and MAC appeared in the early 1970's it took another quarter century for RBAC to thrive and develop robust foundations. RBAC emerged as a result of increasing practitioner dissatisfaction with the then dominant DAC and MAC paradigms, encouraging academic research on RBAC. With the flourish of the distributed systems, RBAC models are more flexible and practical [15].

RBAC is an approach used for advanced access control to restrict system access to authorized users, and is widely deployed in distinct enterprises of all sizes to support information access management. In recent years, some extensions to the authorization as well as the administrative models for RBAC have been adopted [16].

Nevertheless, RBAC is often required to think of various context information in authorization decisions, especially in highly interactive and interconnected environments. Furthermore carry out fine grained policies where permissions are directly assigned to certain individuals in many real-world applications is necessary [17].

Devdatta Kulkarni et al., [10] proposed a CA-RBAC model for pervasive computing applications. Context identification mechanisms can be designed to suggest proper contexts to 
users. The contextual attributes may involve the translation of location, time, proximity, or the executing subject. Due to the increasing demands on the different contextual attributes, this paper adopts CA-RBAC to meet and satisfy the industry needs.

\subsection{Business Process Model and Notation}

An integrated and tool-supported methodology that allows for specifying and executing compliance and security requirements is needed for business process-driven systems [18]. A recent book on business process automation [19] starts with the statement that after almost two decades of extensive research and development in the field. BPMN is a graphical standard informally oriented representation language primarily targeted at domain analysts and supported by many modeling tools [20-22]. Business processes in the early phases of systems development are enumerated and specified by BPMN in a business process model [23].

BPMN has attained some level of adoption among system architects and business analysts as a language for defining business process blueprints for subsequent implementation. A great part of the modeling has to do with the ability to express the different needs of the business as well as to have a notation in which these needs can be described [11]. Despite being a recent proposal, BPMN is already implemented and supported by a number of tools [20]. Therefore, BPMN is an increasingly important standard for process modeling and has enjoyed high levels of attention in business practice [24]. Large global software companies such as IBM [25], Oracle [26], SAP [27] and Unisys supply the BPMN standard solutions [28].

\section{The Design and Implementation of CSIP}

The CSIP includes (1) users, (2) Human-Computer Interface Server (HCIS), (3) Web Service Server (WSS), (4) Content Management Server (CMS), (5) Workflow Server (WFS), (6) Database Server (DBS), (7) Authentication Server (AS), and (8) tools as shown in Figure 1.

\subsection{Users}

Users can utilize various terminal devices that include Personal Computer (PC), notebook, Tablet PC, Personal Digital Assistant (PDA), and smart phone to communicate with CSIP to access the information, tasks, files, etc. This study uses HyperText Markup Language (HTML) and JavaScript to design and implement the client site, so users can utilize various terminal devices to access CSIP.

\subsection{Human-Computer Interface Server}

Users can access CSIP via HCIS which is implemented by using Play framework 1.2.4. with Model-View-Controller (MVC) model. HCIS uses Hibernate technique to build the Object Relational Mapping (ORM) and uses Groovy language to generate the view template. HCIS provides account management, authority management, record management, and message management which are presented as follows. 


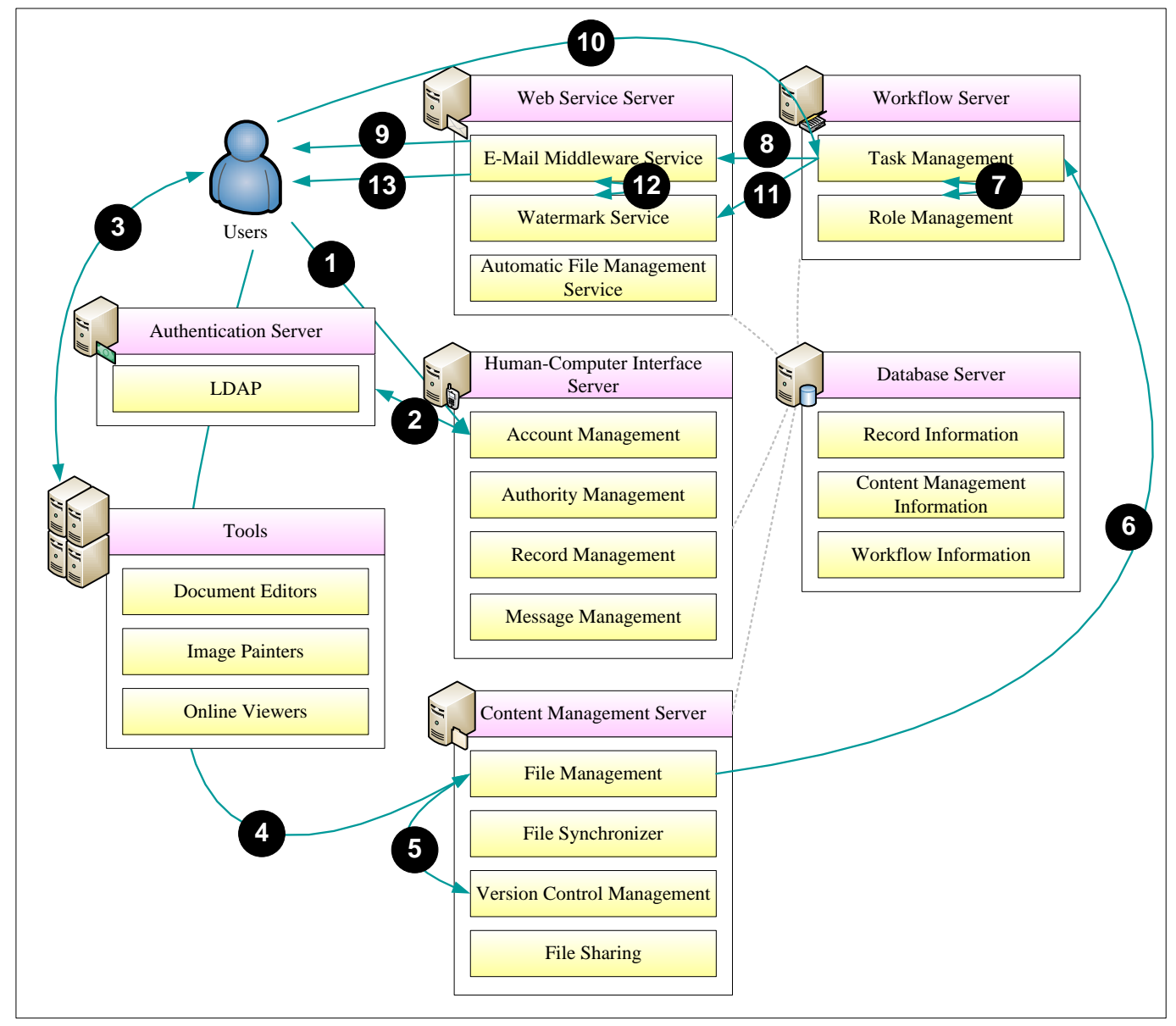

Figure 1. The Architecture of CSIP

3.2.1. Account Management: The account management can offer the user authentication which includes personal data management and password modification. When HCIS receives the login request from user, it communicates with AS via Secure Socket Layer (SSL) to verify the user's account.

3.2.2. Authority Management: The authority management offers project authority, task authority, and file transfer authority.

- Users who are given project authority can access the information of the project.

- Users who are given task authority can view and operate the task.

- Users who are given file transfer authority can transfer the files to their colleagues. Moreover, these files are only accessed by them.

3.2.3. Record Management: The record management can record the creation, read, update, and deletion (CRUD) events from users. For example, John starts and assigns a sign application task to Peter at 08:00, and Peter signs the application at 13:00 and notifies John. Then John can confirm the sign application. All of these events are recorded in log files. 
3.2.4. Message Management: The message management can offer new task notification and provide historical task notifications and task status. For example, Peter can receive the alert immediately when John starts and assigns a task to Peter. Moreover, John can query the task status via HCIS.

\subsection{Web Service Server}

This study uses Tomcat 6.0 framework to implement the WSS and builds the web Application Programming Interfaces (APIs) based on Representational State Transfer (REST) format. The WSS includes E-mail middleware service, watermark service, and automatic file management service which are presented in following subsections.

3.3.1. E-Mail Middleware Service: The external application programs can send parameters and call the APIs which are offered by E-mail middleware service to send E-mail. This study uses Simple Mail Transfer Protocol (SMTP), Secure Socket Layer (SSL), and Transport Layer Security (TLS) to implement the E-mail middleware service which can transfer parameters and files to SMTP server. Then SMTP can send the E-mail to the receivers who are mentioned.

3.3.2. Watermark Service: The watermark service provides APIs for WFS to add watermark into PDF file. This study uses JavaScript Object Notation (JSON) to communicate with the PDF process components for processing PDF file. When user wants to add watermark for sign application, WSS can communicate with CMS and add watermark into the files.

3.3.3. Automatic File Management Service: The automatic file management service offers the APIs to external application programs for file management which includes upload, update, remove, download functions. This study uses Java and JavaScript to implement the automatic file management service and uses JSON to send and receive the data and objects.

\subsection{Content Management Server}

The CMS is a content and resource storage center and combines with File Transfer Protocol (FTP), Web Distributed Authoring and Versioning (WebDAV), Network File System (NFS), and Content Management Interoperability Services (CMIS) for resource access. This study uses Alfresco framework which is an open-source system based MVC model to implement the CMS.

3.4.1. File Management: The file management can provide creation, modification, copy, and move functions. Moreover, the file management also groups users into managers, collaborators, contributors, and consumers. The administrator can add and update the role groups in accordance with the business requirement. For example, Peter can create a new project, and he is the project manager. He invites John as a contributor, Mary as a contributor, and Alice as a consumer. Peter creates a new file which can be revised and copied by John. John creates a new file which can be revised, removed, copied, and moved by Peter. Alice only can read.

3.4.2 File Synchronizer: User can drag and drop the file icons to upload these files to CMS, and the version information of file can be recorded simultaneously. User also can install an application program which can support Microsoft Windows and Apple Mac OS to synchronize the client and server. 
3.4.3. Version Control Management: The version control management can provide not only the newest version file but also the old version files. When the file is synchronized or updated, the difference between versions can be recorded and compared by version control management.

3.4.4. File Sharing: Users can get the file Uniform Resource Locator (URL) link from CMS and share the link to their colleagues. Their colleagues can get the link and download the file after getting authority. For business process, a file URL link can be shared with the user who handles the next task after completing the current task. For example, John starts and assigns a workflow task to Peter, Peter can get file URL link after receiving the task notification.

\subsection{Workflow Server}

The business processes can be built in the WFS which is implemented by using Bonita framework 5.9.1. This study uses BPMN 2.0 to build the workflows of business process, and the workflows include pools, lanes, tasks, roles, gateways, connector, and messages. The tasks, roles, messages of task, user actions are predefined in each workflow. WFS offers task management and role management which are presented as follows.

\subsubsection{Task Management}

The WFS can offers task management to start the task in accordance with the order and logic of workflow and to communicate with the connectors which can be used on ready state, start state, and finish state for logic control. The kinds of tasks operation are classified as human task, service task, script task, sending task, and receiving task presented as follows.

(1) A human-computer interface (i.e., web page) is assigned to user for view and operation when a human task starts. User can use his account to login CSIP and explore the content of web page. When user inputs some parameters and submits, the human task is finished and activates the next task of the workflow. For implementation, this study uses HTML and JavaScript to develop human task.

(2) The service task and script task which can be used to build the logic instruction for workflow are implemented by using Java language.

(3) The sending task and receiving task used to send and receive the messages between the tasks of workflow, especially across the different workflows and pools.

\subsubsection{Role Management}

The kinds of users and roles are classified as user, user role, and user group which are presented as follows. Therefore, this study proposes and designs the CA-RBAC model based on the user and role classification to manage the authority of role.

For the configuration of user and role objects, the user and role (e.g., employee, manager, and agent) in workflow are defined in accordance with the business process. The relationships between users and roles are permitted as many-to-many. For example, John is an employee, and he also is Peter's agent. The user group can be created in accordance with the business process to create the group for the related users. For example, a task which is involved by all employees at the production department, and a user group can be created for the employees at the production department. Then the task can be assigned to the user group.

This study also considers the context information which includes time, location, and environment information to enhance RBAC and adjust the authority of user role dynamically. For implementation, the logic gateways and connectors are added in the tasks and workflows 
for adjusting the authority dynamically. The right information can be accessed by the right user in CSIP based on CA-RBAC. For example, John can set Peter as his agent during August 2013. Peter cannot possess the authority of John's agent after August 2013.

A case study of CA-RBAC is given to show that the agent algorithm automatically adjusts and assigns the authority of user role access privilege. Context requirements must be set to dynamic permission by the right user to access information in CSIP based on CA-RBAC. The components and CSIP Agent Algorithm are described as Table 1. Permission Agent Element (PAE) includes has agent (ha), userid (uid), agented (aid), agentStarttime (ast), agentEndtime (aet) and checkSigned (cs). Inform Agent Element has agented (aid) and agentEmail (aem).

\subsection{Database Server}

For implementation, this study uses MySQL Server 5.5 technique to implement the DBS which includes HCIS data, CMS data, WFS data, connection module, and control module. The server also offers the web services properties to store and access, and it is a powerful application tool to provide reliable information for collaboration.

\subsection{Authentication Server}

This study designs an AS to integrate the CSIP and the existed servers in company for single sign-on (SSO). AS is implemented with Light-weighted Data Access Protocol (LDAP) on Ubuntu 12.04 to build the user's accounts and information. When HCIS receives the login request from user, it can send the request to AS via SSL to verify the user account.

\subsection{Tools}

The CSIP which is an open platform can provide APIs to interact with external systems and tools. The tools (e.g., document editors, image painters, and online viewers) can be embedded and built in CSIP easily for user operation.

Table 1. CSIP Agent Algorithm

\begin{tabular}{|c|c|}
\hline Input & User $U$, Notification List $N L$, Current context $C$ \\
\hline Output & IAE \\
\hline Procedure & $\begin{array}{l}\text { begin } \\
\text { IAE } \leftarrow \text { Null } \\
\text { while ha }=\text { true } \\
\text { If } c s=\text { false } \\
\text { then } \\
\text { read current context } C \\
\mathrm{PAE} \leftarrow \text { uid, aid, ast, aet } \\
\text { while currenttime }>\text { aet } !=\text { true } \\
\text { if currenttime }<\text { ast } \| \text { currenttime }>=\text { ast } \\
\text { then } \\
\text { while currenttime }<\text { ast } \\
\text { currenttime } \leftarrow \text { currenttime }+1 \\
\text { end while } \\
\text { insert aid and ae to NL } \\
\text { IAE } \leftarrow \text { PAE, NL } \\
\text { end if } \\
\text { end while } \\
\text { end if } \\
\text { end while } \\
\text { end }\end{array}$ \\
\hline
\end{tabular}




\section{A Case Study}

For OA, a case study is given to explain the roles, workflows, and messages in CSIP (shown in Figure 1). There are two users who play the different roles in this case: Peter is an employee, and John is Peter's manager. Peter generates an authorization request via online system in this case. The detail of system flows is described as follows.

Step 1-2: Peter can use various devices (ex., PC, smart phone, or tablet PC) to connect with HCIS and input his account and password via SSO system. Then the account management service sends the account and password to AS. After authenticating Peter's account, AS will response a token which can be used to connect to other servers without login process.

Step 3: Peter can use the tools to edit his documents via online system. After editing documents, Peter can output the documents as PDF format files for review processes.

Step 4-5: After generating PDF files, Peter can upload these files to the CMS via the file management service. Then CMS activates the version control management service to record the file version and $\log$ the status of file modification.

Step 6: After the file management processes, the CMS activates the task management service in WFS to initiate the OA task.

Step 7-10: The CMS can check and assign the task to the correct role and user. In this case, the task is assigned to manager (i.e., John). Therefore, the CMS activates the E-mail middleware service to send E-mail which notifies John that an unauthorization job is required to be approved by him. When John receives the E-mail, he can click the URL link to login the WFS. John can review and approve the documents via online system (shown in Figure 2).

Step 11-13: Then the WFS activates the watermark service to add the signet watermark into these documents. After adding the signet watermark, the E-mail middleware service sends an E-mail which notifies Peter the all processes of OA are success.

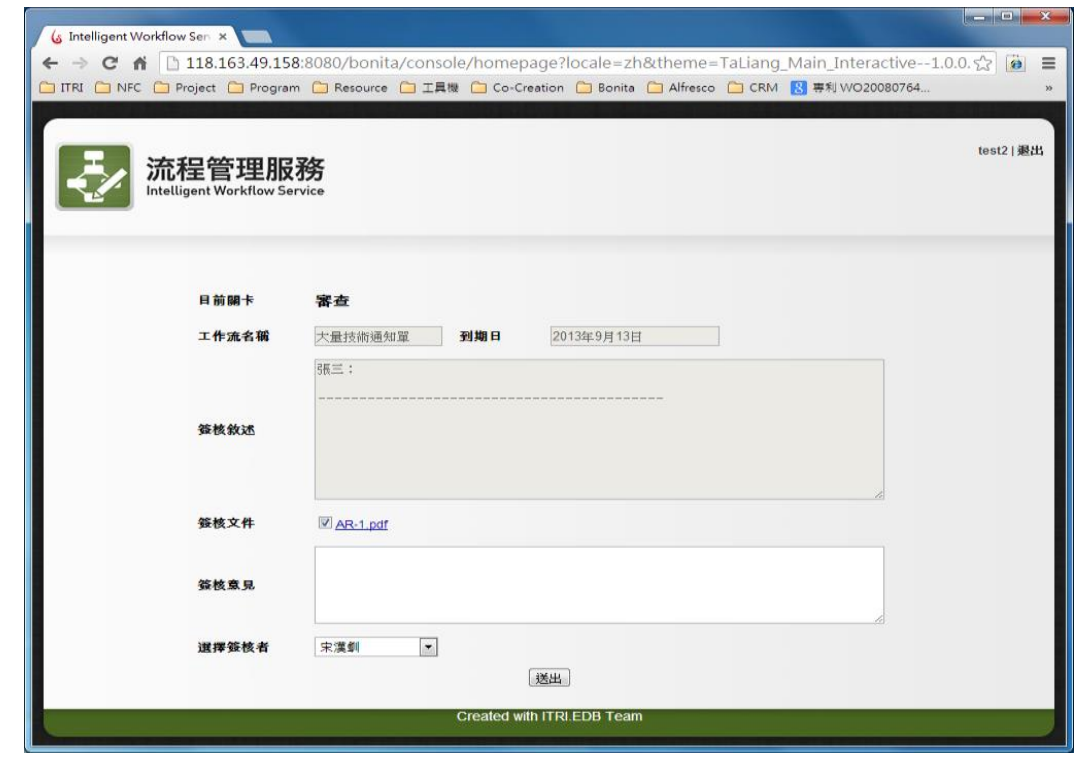

Figure 2. The Approval for Application 


\section{Conclusions}

The effective business process management has received attention recently for improving business process performance and supporting coordination scenarios in collaboration platform. However, the user and role authorities in the existed collaboration platform cannot automatically change in accordance with time and location. Due to the hard diverse process handling with tasks and data separated by enterprise boundaries, this study proposes the CSIP combines the standard BPMN and CA-RBAC model to offer friendly graphical user interface and access control with contextual attributes solution in collaboration environment. A case study of OA is given to present the system flows and authorization mechanisms of CSIP. The CSIP is a practical approach to satisfy the increasing demands on different workflow and contextual attributes in machine tools industry as well. In the future, this platform can be implemented and installed in various companies to support their business process management.

\section{Acknowledgements}

The authors would like to acknowledge the funding agent, the Department of Industrial Technology/MOEA, for supporting this study by the project- Gamification Technologies Enabling Mobile Coupon Recommendation Services (102-EC-17-A-0105-0337).

\section{References}

[1] M. Cao, Q. Zhang, "Supply Chain Collaboration: Impact on Collaborative Advantage and Firm Performance", Journal of Operations Management, vol. 29, no. 3, (2011), pp. 163-180.

[2] R. K. L. Ko, S. S. G. Lee and E. W. Lee, "Business Process Management (BPM) Standards: a Survey", Business Process Management Journal, Emerald Publishing, vol. 15, no. 5, (2009), pp. 744-791.

[3] S. Y. Lin, C. H. Chen and C. C. Lo, "Currency Exchange Rates Prediction based on Linear Regression Analysis Using Cloud Computing”, International Journal of Grid and Distributed Computing, vol. 6, no. 2, (2013), pp. 1-10.

[4] S. Y. Li, H. Y. Kung, C. H. Chen and W. H. Hsu, "An Efficient RFID Data Processing Scheme for Data Filtering and Recognition", International Journal of u- and e- Service, Science and Technology, vol. 5, no. 2, (2012), pp. 59-76.

[5] Q. Chen, M. Hsu, "Inter-Enterprise Collaborative Business Process Management", Proceedings of 17th IEEE Int. Conference on Data Engineering (ICDE), Heidelberg, Germany, (2001), pp. 253-260.

[6] Google Site, Wikipedia, available: http://en.wikipedia.org/wiki/Google_Sites.

[7] R. S. Sandhu and P. Samarati, "Access Control: Principle and Practice", IEEE Communications Magazine, vol. 32, no. 9, (1994), pp. 40-48.

[8] J. Ho Eom, N. uk Kim, S. hwan Kim and T. M. Chung, "An Architecture of Document Control System for Blocking Information Leakage in Military Information System", International Journal of Security and Its Applications, vol. 6, no. 2, (2012), pp. 109-114.

[9] X. Zhang, S. Oh and R. Sandhu, "PBDM: a Flexible Delegation Model in RBAC", Proceedings of the eighth ACM symposium on Access control models and technologies (SACMAT '03), New York, USA, (2003), pp. 149-157.

[10] D. Kulkarni and A. Tripathi, "Context-Aware Role-Based Access Control in Pervasive Computing Systems", Proceedings of the 13th ACM Symposium on Access Control Models and Technologies (SACMAT'08), Estes Park, CO, USA, (2008), pp. 113-122.

[11] A. Rodr'iguez, E. Fernandez-Medina and M. Piattini, "A BPMN Extension for the Modeling of Security Requirements in Business Processes", IEICE Transactions on Information and Systems, vol. 90-D, no. 4, (2007), pp. 745-752.

[12] B. W. Lampson, "Dynamic Protection Structures", Proceedings of American Federation of Information Processing Societies conference, Las Vegas, Nevada, USA, (1969), pp. 27-38.

[13] D.T.C.S.E.C. (TCSEC), DoD 5200.28-STD Foundations, MITRE Technical Report 2547, (1973).

[14] D. E. Bell and L. J. LaPadula, "Secure Computer Systems: Mathematical Foundations", The MITRE Corp.,vol. 1-111, Bedford, Mass, (1973). 
[15] X. Jin, R. Krishnan and R. Sandhu, "A Unified Attribute-Based Access Control Model Covering DAC, MAC and RBAC", Proceedings of the International Conference on Data and Applications Security and Privacy, , Paris, France, (2012), pp. 41-55.

[16] K. S. Joo and J. W. Woo, "Object-Oriented Analysis and Design Methodology for Secure Web Applications Focused on Role Based Access Control", Proceedings of the 1st International Conference on Advanced Information and Computer Technology (AICT 2013), ASTL, Rome, Italy, vol. 26, (2013), pp. 160-166.

[17] G. Neumann and M. Strembeck, "An Approach to Engineer and Enforce Context Constraints in an RBAC Environment", Proceedings of the eighth ACM Symposium on Access Control Models and Technologies (SACMAT '03), pp. 65-79, New York, USA, (2003).

[18] A. D. Brucker, I. Hang, G. Luckemeyer and R. Ruparel, "SecureBPMN: Modeling and Enforcing Access Control Requirements in Business Processes", Proceedings of the 17th ACM Symposium on Access Control Models and Technologies (SACMAT '12), New York, USA. (2012), pp. 123-126.

[19] A. H. M. ter Hofstede, W. van der Aalst, M. Adams and N. Russell, "Modern Business Process Automation", ISBN 978-3-642-03121-2, Springer, (2010).

[20] C. Ouyang, M. Dumas, A. H. M. ter Hofstede and W. M. P. van der Aalst, "Pattern-based Translation of BPMN Process Models to BPEL Web Services", International Journal of Web Services Research, vol. 5, no. 1, (2008), pp. 42-61.

[21] M. A. López-Campos, A. Crespo Márquez and J. F. Gómez Fernández, "Modelling Using UML and BPMN the Integration of Open Reliability, Maintenance and Condition Monitoring Management Systems: An application in an Electric Transformer System”, Computers in Industry , vol. 64, no.5, (2013), pp. 1524-1542.

[22] P. V. Gorp and R. Dijkman, "A Visual Token-based Formalization of BPMN 2.0 based on In-Place Transformations", Information and Software Technology, vol. 55, no. 2, (2013), pp. 365-394.

[23] R. M. Dijkman, M. Dumas and C. Ouyang, "Semantics and Analysis of Business Process Models in BPMN", Information and Software Technology, vol. 50, no. 21, (2008), pp. 1281-1294.

[24] J. Recker, “Opportunities and Constraints: the Current Struggle with BPMN", Business Process Management Journal, Vol. 16, No.1, pp. 181-201 (2010)

[25] J. M. Diament, A. R. Heching M. Kloppmann, "Dynamic Transfer of Selected Business Process Instance State", U.S. Patent 20120192189, (2012).

[26] A. Aggarwal, V. Saxena and G. Radhakrishnan, "Business Process Diagram (BPD) Component Analysis", U.S. Patent 20100088251, (2010).

[27] R. Day, "Entity Morphing in Meta Model-based Tools", EP Patent 2330501, (2011).

[28] B. S. S. Onggo and O. Karpat, "Agent-based Conceptual Model Representation Using BPMN", Proceedings of the 2011 Winter Simulation Conference, Phoenix, AZ, USA, (2011), pp. 671-682.

\section{Authors}

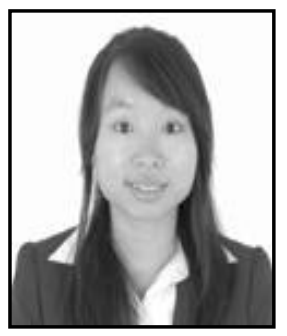

Shu-Ping Lu, received a M.S. degree from Department of Electronic and Computer Engineering at National Taiwan University of Science and Technology, Taiwan, in 2008. She is currently serving as a researcher in Service System Technology Center of Industrial Technology Research Institute. Her interests include web services, information and communication technology, intelligent transportation system, and image processing.

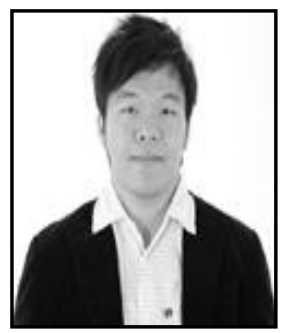

Kuei-Kai Shao, received a M.S. degree from Department of Industrial Engineering and Management at National Taipei University of Technology. He is currently serving as a project manager in Service System Technology Center of Industrial Technology Research Institute. His recent research interests are in web services and software engineering. 


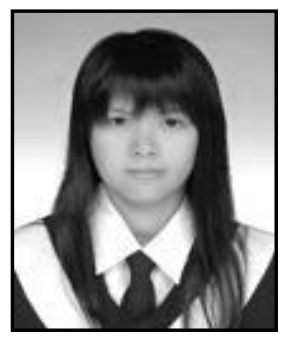

Yu-Nung Chao, received a B.S. degree from Department of Information Management at Chang Gung University of Science and Technology in Taiwan in 2009. She is currently serving as a software engineer in Service System Technology Center of Industrial Technology Research Institute. Her recent research interests are in web services and software engineering.

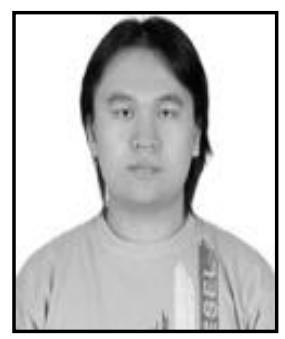

Kuo-Shu Luo, received a M.S. degree from Graduate Institute of Industrial Management at National Central University. He is currently serving as a manager in Service System Technology Center of Industrial Technology Research Institute. His recent research interests are in web services, consumer relationship management and software engineering.

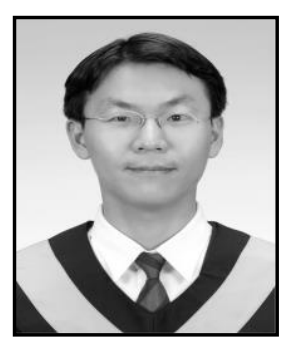

Chi-Hua Chen, received his B.S. degree from Department of Management Information Systems at National Pingtung University of Science and Technology in 2007, a M.S. degree from Institute of Information Management at National Chiao Tung University (NCTU) in 2009, and a Ph.D. degree from Department of Information Management and Finance at NCTU in 2013.

He has published over 100 journal and conference papers. Currently, he is serving as an Editor-in-Chief for IEEE Technology and Engineering Education. He also served as a Guest Editor-in-Chief of Special Issue on "Cloud Computing Technology and Applications" for IEEE Multidisciplinary Engineering Education Magazine, an Area Editor/Associate Editor for International Journal of Communication Networks and Information Security, an Assistant Editor-In-Chief for International Journal of Design, Analysis and Tools for Integrated Circuits and Systems, and a member of editorial board of several international journals. His recent research interests are in cloud computing, cellular networks, data mining, intelligent transportation system, network security, healthcare system, augmented reality, and Elearning system. 
International Journal of Security and Its Applications Vol.8, No.1 (2014) 